\title{
Concept and Analysis for a 1 - 100 PeV Tau Neutrino Observatory
}

\author{
Andrés Romero-Wolf* \\ Jet Propulsion Laboratory, California Institute of Technology \\ E-mail: Andrew.Romero-Wolf@jpl.nasa.gov
}

Washington Carvalho Jr. ${ }^{1,2}$, Jaime Alvarez-Muñiz ${ }^{3}$, José Bazo ${ }^{4}$, José Bellido ${ }^{5}$, Austin Cummings ${ }^{6}$, Alberto Gago ${ }^{4}$, Harm Schoorlemmer ${ }^{7}$, Stephanie Wissel ${ }^{8}$, and Enrique Zas $^{3}$

${ }^{1}$ Department of Astronomy, California Institute of Technology, Pasadena, CA, USA;

${ }^{2}$ Departamento de Física, Universidade de São Paulo, São Paulo, Brazil; ${ }^{3}$ Departamento de

Física de Partículas \& Instituto Galego de Física de Altas Enerxías, Univ. de Santiago de

Compostela, Santiago de Compostela, Spain; ${ }^{4}$ Pontífica Universidad Católica del Perú, Lima,

Perú; ${ }^{5}$ University of Adelaide, Adelaide, SA, Australia; ${ }^{6}$ Gran Sasso Science Institute, School of

Advanced Studies, L'Aquila, Italy; ${ }^{7}$ Max-Planck-Institut für Kernphysik, Heidelberg, Germany;

${ }^{8}$ California Polytechnic State University, San Luis Obispo, CA, USA

\begin{abstract}
The IceCube Neutrino observatory has discovered an astrophysical neutrino flux in the energy range $>1 \mathrm{TeV}$ with their highest energy event $\sim 3 \mathrm{PeV}$. Although IceCube has excellent muon flavor identification, their ability to identify tau neutrinos is highly limited. We present a concept for observing the tau neutrino flux in the $1-100 \mathrm{PeV}$ range, where a flux is guaranteed and its observation would give further support to the astrophysical origin of the IceCube flux. The design of the observatory requires an acceptance $\langle A \Omega\rangle>400 \mathrm{~m}^{2} \operatorname{sr} \times\left(\frac{E}{\mathrm{PeV}}\right)^{1.5}$ to match the $\sim 1 \mathrm{PeV}$ IceCube all-flavor event rate in tau neutrinos provided a $v_{e}: v_{\mu}: v_{\tau}=1: 1: 1$ flavor ratio. The energy dependence of the acceptance is designed to match the IceCube best-fit spectral flux index of $E^{-2.5}$ so the spectrum is evenly sampled in the 1-100 PeV range. We present a backgroundfree implementation with pointing resolution $<1^{\circ}$ to begin the era of high energy tau neutrino astronomy.
\end{abstract}

Acknowledgements: Part of this work was carried out at the Jet Propulsion Laboratory, California Institute of Technology, under a contract with the National Aeronautics and Space Administration. A. R.-W. thanks the Caltech/JPL President's and Director's Research and Development Fund. W.C. thanks grants \#2015/15735-1 and \#2018/18876-3, São Paulo Research Foundation (FAPESP). Copyright 2019. All rights reserved.

36th International Cosmic Ray Conference -ICRC2019-

July 24th - August 1st, 2019

Madison, WI, U.S.A.

* Speaker. 


\section{Introduction}

We present a concept for a deep valley $\tau$ neutrino detector designed for the 1-100 PeV band. The concept of detection is illustrated in Figure 1. Neutrinos propagating in rock have an interaction length of thousands of kilometers for 1-100 PeV energies. A $\tau$ neutrino can interact in rock to produce a $\tau$ lepton, transferring $\sim 80 \%$ of its energy. The $\tau$ lepton has a range of $50 \mathrm{~m}-5 \mathrm{~km}$ for the corresponding energy range of $1-100 \mathrm{PeV}$ before it decays. If the interaction occurs within this distance from a deep valley, the $\tau$ lepton is likely to exit the rock into the air. Within this range, the $\tau$ lepton may decay in air. Roughly $50 \%$ of the $\tau$-lepton energy (on average) goes into particles that produce extensive air showers. The longitudinal profile of the electromagnetic component of these air showers has a characteristic length of $\sim 10 \mathrm{~km}$, and a diameter of $\sim 200 \mathrm{~m}$ near shower maximum. A deep valley with mountain separations comparable to this length scale and an array of small water-Cherenkov detectors $\left(\sim \mathrm{m}^{3}\right.$ volume) separated by $\sim 100 \mathrm{~m}$ on the mountain slope, would be highly sensitive to these $\tau$-lepton air showers. The deep valley topography also provides a significant increase to the geometric acceptance compared to a flat ground array sensitive to earth-skimming air showers.

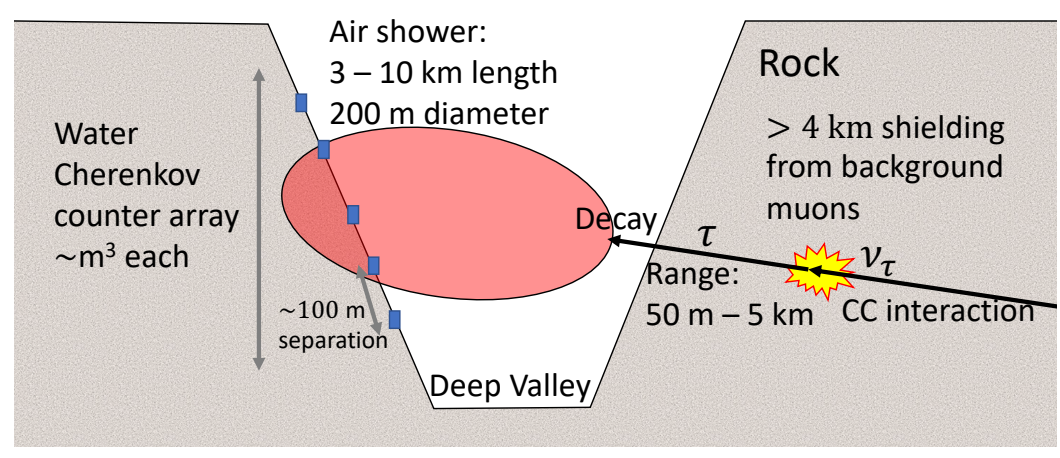

Figure 1: Detector concept schematic. See text for detailed description.

\section{Key Science Goals and Objectives}

The science objectives of the deep valley observatory concept are summarized in the table below.

\begin{tabular}{|c|c|c|}
\hline Objective & Physical Parameters & Observatory Requirements \\
\hline $\begin{array}{l}\text { (01) Determine whether high } \\
\text { energy neutrino sources } \\
\text { continue to accelerate particles } \\
\text { above } 10 \mathrm{PeV} \text {. }\end{array}$ & $\begin{array}{l}\text { Sensitivity of } \geq 5 \sigma \text { to the } \tau \\
\text { component of the flux } \\
\text { extrapolated from IceCube } \\
\text { data for energies } 1 \text { - } 100 \\
\text { PeV }\end{array}$ & $\begin{array}{l}\text { Diffuse } \tau \text { neutrino flux acceptance } \\
\langle A \Omega\rangle \geq 400 \mathrm{~m}^{2} \mathrm{sr} \times E_{\mathrm{PeV}}^{3 / 2} \text { between } 1-10 \mathrm{PeV} \\
\text { and }>10 \text { times IceCube between } 10-100 \mathrm{PeV} \text {. } \\
\text { Integrated sky coverage }>0.5 \mathrm{sr} \text {. }\end{array}$ \\
\hline $\begin{array}{l}\text { (02) Constrain the astrophysical } \\
\text { nature of the neutrino flux } \\
\text { between } 1-10 \mathrm{PeV} \text { by } \\
\text { measuring the } \tau \text { component. }\end{array}$ & $\begin{array}{l}\text { Sensitivity to the diffuse } \tau \\
\text { neutrino flux at energies } \\
\text { between } 1-10 \text { PeV with } \\
\text { efficient flavor } \\
\text { identification. }\end{array}$ & $\begin{array}{l}\text { Energy resolution: neutrino } \Delta E / E \leq 1.0 \text {, air } \\
\text { shower } \Delta E / E \leq 0.8 \text { (both } 1 \sigma \text { ) } \\
\text { Tau air shower direction resolution } \leq 1^{\circ} \text {. } \\
\text { Tau neutrino flavor identification }>95 \% \\
\text { confidence per event. }\end{array}$ \\
\hline $\begin{array}{l}\text { (03) Constrain the particle } \\
\text { acceleration potential of point } \\
\text { source transients observed with } \\
\text { multi-messenger probes. }\end{array}$ & $\begin{array}{l}\text { Point source flux of } \tau \\
\text { neutrinos as a function of } \\
\text { energy. }\end{array}$ & $\begin{array}{l}\text { Point source effective area } \\
\langle A\rangle \geq 300 \mathrm{~m}^{2} \times E_{\mathrm{PeV}}^{3 / 2} \text { (peak) with instantaneous } \\
\text { sky coverage }>0.1 \mathrm{sr} \text {. }\end{array}$ \\
\hline
\end{tabular}


Objective $\mathrm{O} 1$ is motivated by the question of whether the sources of high energy neutrinos have a cutoff at $\sim 6 \mathrm{PeV}$, as suggested in some recent studies [1]. Even if this is the case, the cosmogenic neutrino flux is expected to dominate near $100 \mathrm{PeV}$. In Figure 2, we show the extrapolated IceCube flux [2] compared to models of cosmogenic neutrino fluxes. The Kotera 2010 [3] range of fluxes assume cosmic ray compositions and source energy cutoffs generally more optimistic than fits to the Pierre Auger Observatory data [4], while Romero-Wolf \& Ave 2018 [5] use constraints from Auger data. These models represent two extremes of what the cosmogenic neutrino flux could be and, in both cases, the flux is comparable to the extrapolated IceCube flux for energies approaching $100 \mathrm{PeV}$.

Objective $\mathrm{O} 2$ is motivated by the lack of unambiguous detections of high energy $\tau$ neutrinos to date. Observations in the $1-10 \mathrm{PeV}$ energy band would be complementary to IceCube in that the deep valley observatory is sensitive primarily to $\tau$ neutrinos.

Objective $\mathrm{O} 3$ is directly motivated by multi-messenger observations. The deep valley observatory sensitivity is focused in a region of $\sim 30^{\circ}$ elevation angle and $120^{\circ}$ azimuth (see next section), which potentially enables a higher point source sensitivity. The region of the sky covered depends on the latitude of the observatory, which can in principle be placed anywhere there is a deep valley of appropriate dimensions (Figure 1).

In addition to the objectives stated above, we are investigating additional science goals that could be achieved with a deep valley air shower array. The unique topography could be used to improve our understanding of air shower physics and to improve the measurement of the depth of shower maximum with water-Cherenkov detectors by detecting vertical showers and measuring the shower size at different slant depths. We are looking into the possibility of discriminating photons from hadrons and contribute to gamma-ray observations at the $\mathrm{PeV}$ region and beyond. It may also be possible to characterize the cosmic-ray anisotropy at PeV energies with new measurements from a latitude corresponding to the proposed observatory site in the Colca Valley, Peru. This would overlap with the HAWC and IceCube sky exposures [6], providing complementary information to characterize the anisotropy and further constrain its origin.

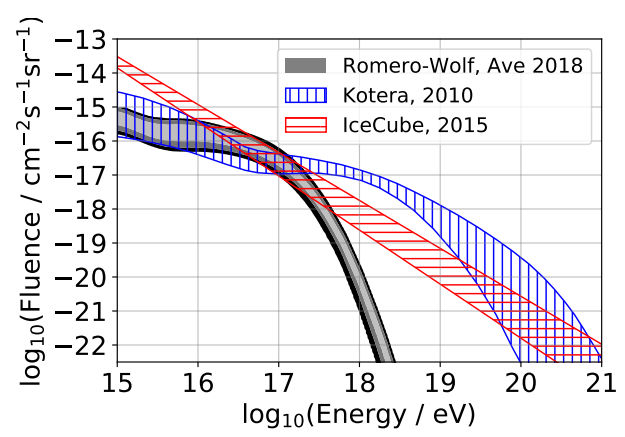

Figure 2: The IceCube neutrino flux $(\propto$ $E^{-2.5}$ ) [7], extrapolated to high energies, compared to predictions of the cosmogenic neutrino flux of Kotera 2010 [3] and RomeroWolf \& Ave 2018 [5] In either case, the flux of cosmogenic neutrinos is comparable to the extrapolated IceCube flux at energies approaching $100 \mathrm{PeV}\left(10^{17} \mathrm{eV}\right)$. Figure adapted from [5].

\section{Observatory Requirements}

Diffuse flux acceptance: At $\sim 1 \mathrm{PeV}$ energy, the IceCube acceptance for $\tau$ neutrinos is $\langle A \Omega\rangle \simeq 130 \mathrm{~m}^{2} \mathrm{sr}$ [8]. Since the detector presented here would be primarily sensitive to $\tau$ neutrinos and not other flavors, we target a $v_{\tau}$ acceptance of $400 \mathrm{~m}^{2} \mathrm{sr}$, which is approximately three times the single-flavor acceptance of IceCube at $1 \mathrm{PeV}$. The IceCube single power-law flux for cascade events is $\propto E^{-2.5}[7,2]$, which drives the requirement that the acceptance be proportional to $E^{1.5}$. 
The size and number of detectors on this observatory has been designed around the requirement that $\langle A \Omega\rangle \geq 400 \mathrm{~m}^{2} \mathrm{sr}\left(\frac{E}{\mathrm{PeV}}\right)^{1.5}$ at energies between 1-10 PeV, for objective $\mathrm{O}$ 2, and that the acceptance be at least an order of magnitude greater than IceCube in the 10-100 PeV energy band for objective O1. The expected performance, based on simulations described in the next subsection, is shown in Figure 3.

The probability of $\tau$ lepton production from $v_{\tau}$ interactions in rock that exit to the atmosphere provides the desired $E^{1.5}$ dependence on the acceptance [9]. The decay of $\tau$ leptons exiting the rock will produce air showers which can be detected with conventional particle counters [10, 11]. This technique preferentially detects $\tau$ neutrinos due to the combination of the decay lifetime and energy loss of the $\tau$ leptons produced in a charged current interaction. Electrons produced by electron neutrinos interact in rock lose energy almost immediately. Muons produced by muon neutrinos can exit into the atmosphere but their detection is suppressed by the long muon lifetime and the low probability of interactions that result in significant energy transfers in the atmosphere. The differential event rates $(\sim 7$ events per year total is shown in the right panel of Figure 3 .

Energy Resolution: A significant part of the energy resolution error budget is dominated by the neutrino interaction and $\tau$-decay process. The average energy transfer to the lepton in a charged current interaction that exits the rock is $\sim 80 \%$. Using the results of [9], the exiting $\tau$ lepton has an energy spread of $\sim 14 \%$ around the most probable energy. For the purposes of this discussion, uncertainties and resolution values are $1 \sigma(68 \%$ confidence) unless otherwise stated.

The fraction of energy transferred to particles that produce showers (electrons and hadrons) from a $\tau$-lepton decay has an average value of $55 \%$ with an uncertainty of $60 \%$, based on Pythia simulations [12] of tau lepton decays at relativistic energies. The $\tau$-lepton decays that produce hadrons are dominant (64.8\%) and the next dominant mode is a decay that produces an electron $(17.85 \%)$. The deep valley observatory is not designed to distinguish between electromagnetic and hadronic air showers, although this could be a considered as a future upgrade by adding the
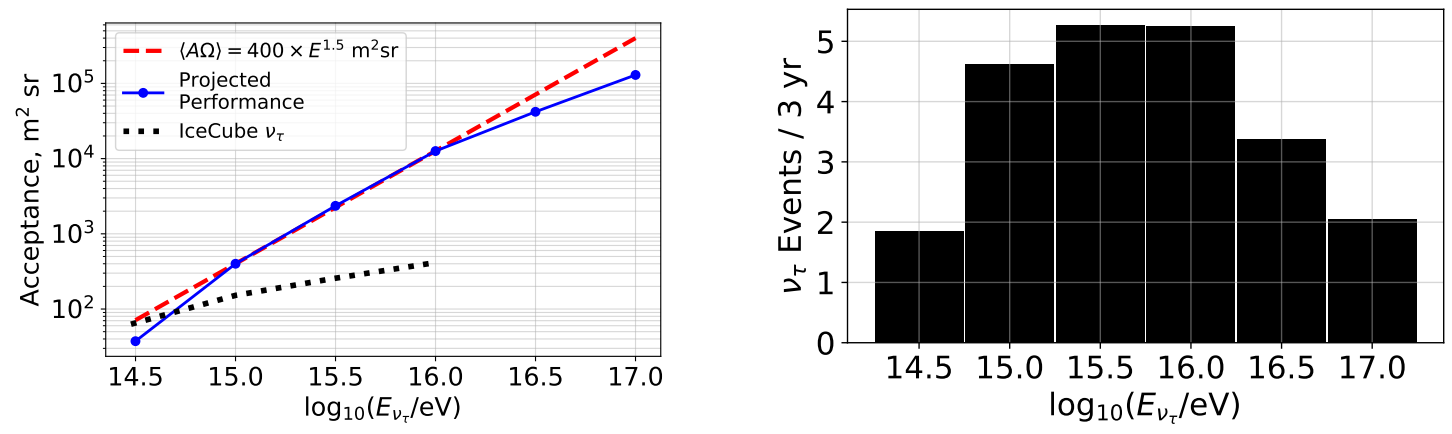

Figure 3: Left: projected performance for the acceptance as a function of energy of an array of $\mathrm{m}^{3}$ waterCherenkov detectors with $150 \mathrm{~m}$ separation and 22,000 detectors. The reduced acceptance at lower energies $(<1 \mathrm{PeV})$ is due to the air showers being too small to trigger the array on the other side of the valley while at high energies $(>10 \mathrm{PeV})$ the loss in sensitivity is due to the $\tau$-lepton range exceeding the dimensions of the valley. The $\tau$ neutrino IceCube acceptance [8] is shown for comparison. Right: the total expected rate with this acceptance on an extrapolated IceCube flux is 21 events per 3 years of operation with a peak at $3 \mathrm{PeV}$. We have used the $\propto E^{-2.5}$ neutrino flux fit from cascade events of [2]. Using the $\propto E^{-2.2}$ fit to the muon neutrino flux from [1] would lead to higher event rates. 
capability to estimate the muon content of the air shower. The remaining decay modes produce a muon, which we consider undetectable.

The combined uncertainties of the energy transferred from the neutrino to the $\tau$ lepton and the $\tau$ lepton decay summed in quadrature is $\sim 62 \%$. If the shower energy resolution is $\Delta E / E \leq$ $80 \%$, then this would achieve the target $\tau$ neutrino energy resolution $\Delta E / E \leq 100 \%$ (also summed in quadrature). Air shower arrays typically perform better than $40 \%$ energy resolution $[13,14]$. However, these observatories can assume a constraint on where the first interaction of a cosmic ray occurs in the atmosphere. For $\tau$ decays in a deep valley, the position of a $\tau$ decay is not nearly as well constrained since it depends on the lifetime of the $\tau$, which ranges from $50 \mathrm{~m}$ at $1 \mathrm{PeV}$ and $5 \mathrm{~km}$ at $100 \mathrm{PeV}$. Initial results, using an estimate of the total estimated number of particles in tanks with signal equivalent to 3 or more particles, constrain the shower energy to within an order of magnitude but with long tails in the distribution. The estimated uncertainties provided are only for mono-energetic neutrinos and the ability to reconstruct the energy given a $\tau$ neutrino spectrum is pending. Note, however, that the expected event rate as a function of energy is relatively flat (right panel of Figure 3) which reduces the uncertainties due to spillover in spectral bins. Systematic uncertainties in energy have not been included in this discussion. A study aimed at improving the shower energy resolution using fits to the shower profiles and timing between signals sampled with the detector array will be the subject of future work.

Pointing Resolution: The pointing resolution requirement of $\sigma_{\theta} \leq 1^{\circ}$ was identified as sufficient to enable astronomical observations in [15]. Air shower arrays typically achieve $\sim 1^{\circ}$ pointing resolution $[16,17,18,19,20]$. This is enabled by the timing resolution of each detector and their separation $D$ via the relation $c \sigma_{\tau} \leq \sqrt{2} D \cos \theta \sigma_{\theta}$, where the $\sqrt{2}$ factor comes from the fact that at least 2 pairs of detectors are involved in a triggered event. For $\mathrm{D}=150 \mathrm{~m}$ (see Observatory Design section below) and a mountain slope of $35^{\circ}$, the timing uncertainty required is $\sigma_{\tau} \leq 7 \mathrm{~ns}$ in the horizontal direction where the acceptance peaks. Photomultiplier tubes (PMTs) with $\sim$ ns time resolution are common and it is expected that this requirement could be satisfied.

Flavor Identification: This array has been designed to be primarily sensitive to $\tau$ neutrinos by virtue of $v_{\tau}$ interactions and $\tau$-lepton propagation in rock. However, there is the potential of having $\lesssim 1$ background event for every $20 \tau$ neutrino events (see Backgrounds section below). This tolerance still satisfies the $5 \sigma$ sensitivity required by objective $\mathrm{O} 1$.
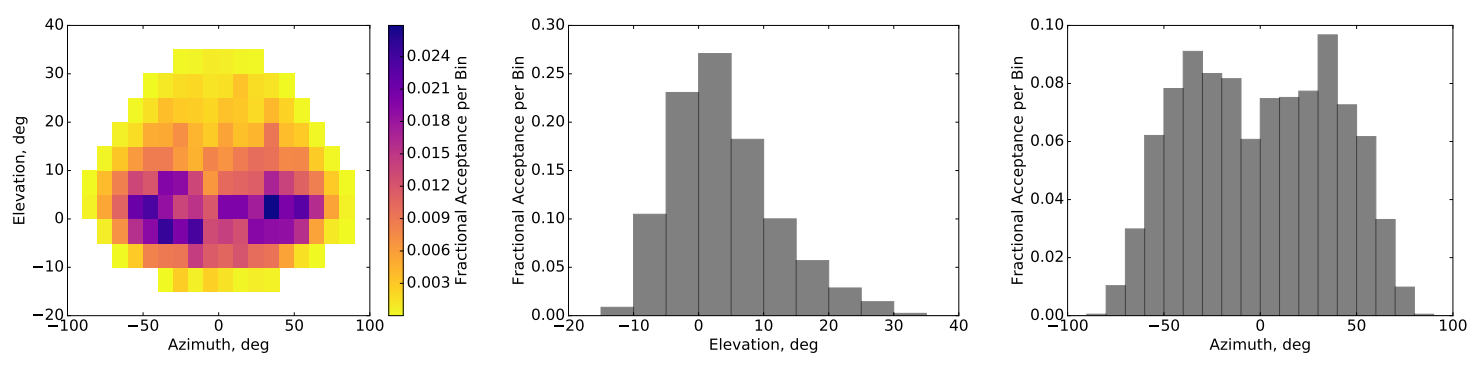

Figure 4: Left: the fractional acceptance (normalized to unity) as a function of particle arrival directions that trigger the array. Middle: the elevation angle projection from the figure on the left. Right: the azimuth angle projection from the figure on the left. The majority of particles arrive near the horizontal direction. The span in azimuth is limited by the decay distance and detector density. 
Point Source Effective Area: While this array was designed by optimizing for the diffuse flux acceptance, it is expected to have a sizeable point source effective area. The field of view is restricted to the range of elevation angles subtended by the mountain facing the detector array $\left(\sim 25^{\circ}\right)$ and azimuthally by the air shower longitudinal profile $(\sim 10 \mathrm{~km})$ corresponding to $\pm 60^{\circ}$ (see Figure 4). Based on the behavior of the diffuse acceptance flux, we have a goal of achieving a point source effective area of $\langle A\rangle \gtrsim 300 \mathrm{~m}^{2} \times(E / \mathrm{PeV})^{1.5}$. This would roughly match the IceCube effective area of $\sim 200 \mathrm{~m}^{2}$ at $1 \mathrm{PeV}$ [21], and exceed it by more than an order of magnitude at 100 $\mathrm{PeV}$.

Sky Coverage: The sky coverage can be optimized to cover a wide region, by spreading the detectors across various valley orientations and locations across the world, or over a narrow region optimizing for point source sensitivity, by clustering the detectors over a long valley that is roughly oriented towards the same direction in the sky. The Colca Valley site (Peru) used for our current study has regions that would point North/South as well as East/West. With the $\gtrsim 2 \mathrm{~km}$ altitudes over which detectors are populated, it is expected that the detector would cover $>0.1 \mathrm{sr}$ instantaneous field of view (see Figure 4). With the rotation of the Earth, the integrated coverage is expected to cover $>0.5 \mathrm{sr}$. It is possible to consider more than one site and detector layout to increase sky coverage or point to specific sources of interest. These trades will be considered in future stages of the observatory design layout.

Backgrounds: In order to achieve the $5 \sigma$ sensitivity required by objective 1 , the backgrounds need to be suppressed to $\lesssim 1$ event per 3 years, based on the expectation of $\sim 20$ events per 3 years. We have considered four main sources of background.

The first source of background considered is due to random coincidences of small showers and vertical muons mimicking a $\tau$ lepton air shower coming from the other side of the valley. The rate of small showers and muons for an array of the size proposed here is in the scale of $\mathrm{kHz}$. Strategies to minimize this background are under investigation. The current trigger design requires three tanks with the equivalent signal of 3 particles each, but also requires that the summed signal of triggered tanks be greater than the equivalent of 30 particles since there is typically one tank closest to the shower core. Additional discrimination strategies include the use of shower profile fits and signal timing constraints.

Second is the directional reconstruction uncertainty of cosmic ray air showers near the skymountain boundary that would appear to point to the mountain facing the detector. With $\sigma_{\theta}=1^{\circ}$ resolution, this background is efficiently suppressed to $<0.1$ events per 3 years by setting a cut on events that have a direction more than $8^{\circ}$ below the sky-mountain boundary. Figure 4 shows the fractional acceptance at $1 \mathrm{PeV}$ as a function of direction. The sky-mountain boundary is at an elevation angle of $\sim 35^{\circ}$ and cutting out $8^{\circ}$ below it is of minimum impact to the acceptance.

Third is the high energy muons produced by cosmic ray air showers [22] behind the mountain that would propagate through to produce an air shower in the valley. If a muon interacts with air via nuclear bremsstrahlung, a sizeable energy transfer to a photon producing an electromagnetic air shower may occur (although the probability is estimated to be $<0.4 \%$ based on AIRES simulations). The energy loss of muons in rock combined with the expected spectrum of high energy muons results in this background being suppressed below the detector threshold for trajectories of $>4 \mathrm{~km}$ of rock. The regions with $<4 \mathrm{~km}$ of path length through rock are already cut out by the cosmic ray air shower cut described in the paragraph above. 
The final source of background considered is from prompt muon neutrinos produced in air showers [22]. Muon neutrinos propagating in rock can undergo a charged current interaction that produces a muon. The range of the muon is in the scale of kilometers meaning that the production of muons per neutrino exiting the rock into the atmosphere is greater than that of $\tau$ leptons. However, the muon lifetime is much longer and it is highly unlikely to decay before reaching the detector. There is still a small probability $(<0.4 \%)$ that an energetic muon can transfer a significant fraction of its energy to a photon via a nuclear brehmstrahlung interaction resulting in an electromagnetic air shower. Preliminary estimates result in an expected value of 1 muon neutrino background event per $20 \tau$ lepton events for energies $\geq 1 \mathrm{PeV}$. More accurate bounds, which are expected to be lower, are currently being studied with more detailed simulations.

\section{Observatory Design}

The approach to observatory design was to identify a deep valley first and then optimize the array design to achieve the required diffuse flux acceptance while minimizing the number of stations. The deep valleys applicable for this concept need to have a width of 3-10 km, driven by the longitudinal profile of the electromagnetic component of air showers, and km-scale depth for efficient use of a detector array. There also needs to be $\sim 100 \mathrm{~km}$ length of mountain face (both sides can be used) to achieve the required acceptance. Since this concept relies on the use of waterCherenkov detectors, it is preferable to have a source of water nearby with existing infrastructure to reduce the cost of construction. Although this list is by no means exhaustive, we have considered the following four of the world's deepest valleys. These are Hells Canyon in the United States of America, Yarlung Tsangpo Grand Canyon in China, Cotahuasi Canyon and the Colca Canyon, both in Arequipa, Peru.

The Colca Canyon has a river running at its base, road access, and nearby towns. These features make it an attractive site. The Colca Canyon has regions with up to 3,270 $\mathrm{m}$ depth with $\gtrsim 100 \mathrm{~km}$ length of mountain face. In Figure 5 we show an approximate cross section of the canyon located near $15^{\circ} 38^{\prime} 35^{\prime \prime} \mathrm{S} 72^{\circ} 08^{\prime} 55^{\prime \prime} \mathrm{W}$ that we used as the fiducial geometry for the results in this white paper. A more detailed optimization study using the topography along the valley will be the subject of a future study.

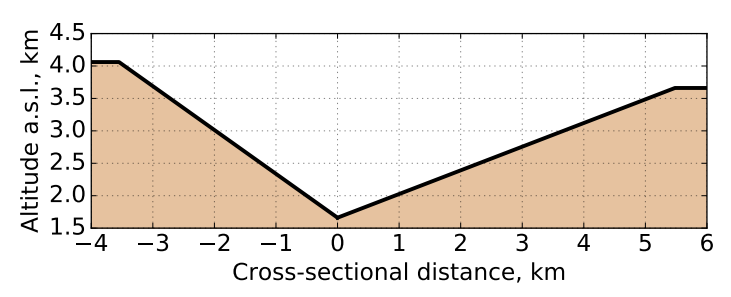

Figure 5: A cross section of the Colca Valley near $15^{\circ} 38^{\prime} 35^{\prime \prime} \mathrm{S} 72^{\circ} 08^{\prime} 55^{\prime \prime} \mathrm{W}$. The distance between the mountain faces is $\sim 4.5 \mathrm{~km}$ at half the valley depth. For the simulation results presented, the left side of the valley was populated with a detector array.

Detector Array Design: Given that a large area has to be covered to reach the required acceptance, we have taken into consideration minimizing the size and number of detectors needed. Based on the density of photons and charged particles in the shower, a water-Cherenkov detector with cross-sectional area of $\sim \mathrm{m}^{2}$ and $\sim \mathrm{m}^{3}$ volume. The trigger requires 3 tanks with a time over threshold trigger requiring the equivalent of at least three particles entering each tank, similar to Auger [23]. We add the requirements that the sum of signals in tanks exceeding the 3 particle equivalent is at least the equivalent of 30 particles to reduce backgrounds from random coincidence. This is based on the expectation that tanks near the air shower core will have a significant 
signal. We ran a simulation to determine the minimum number of tanks needed (see Figure 6) and arrived at 22,000 of them with $150 \mathrm{~m}$ spacing in a triangular grid on the mountain slope. There is a trade between the number of tanks and their size. However, since these detectors have to be placed on steep mountain slopes (up to $35^{\circ}$ ) it is important to keep the size of the tanks small to facilitate deployment.

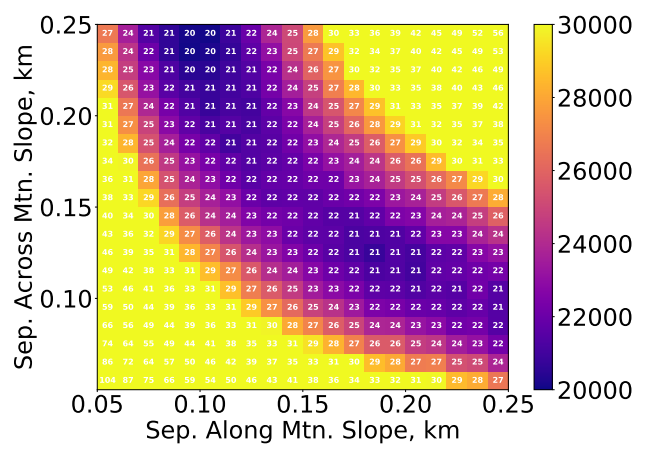

Figure 6: Search for the minimum number of stations, shown in a color scale that saturates above 30,000 , as a function of separation along and across the mountain slope, required to obtain $\langle A \Omega\rangle=400 \mathrm{~m}^{2}$ sr at $1 \mathrm{PeV}$. The number of stations (in thousands), corresponding to the color scale, is displayed in white text in each bin. There is a trough of values that achieve the target acceptance with $\sim 22,000$ stations (with statistical fluctuations). The optimal separation is $\sim 150 \mathrm{~m}$ in both directions.

\section{References}

[1] IceCube Collaboration, C. Haack and C. Wiebusch, PoS(ICRC2017)1005, (2017).

[2] IceCube Collaboration, Proceedings of Science, PoS(ICRC2017)968, (2017)

[3] K. Kotera, D. Allard, and A. Olinto, Jour. of Cosmo. and Astropart. Phys. 10, (2010), 013.

[4] The Pierre Auger Collaboration, Jour. of Cosmo. and Astropart. Phys., 04, 038, (2017).

[5] A. Romero-Wolf, and M. Ave, Jour. of Cosmo. and Astropart. Phys. 7, (2018), 025.

[6] HAWC and IceCube Collaborations, Proceedings of Science, PoS(ICRC2017)539, (2017)

[7] M.G. Aartsen, et al., The Astrophysical Journal, 809, 98, (2015)

[8] IceCube Collaboration, M. G. Aartsen et. al., Science 342 (2013) 1242856.

[9] J. Alvarez-Muñiz et al., Phys. Rev. D, 97, 023021, (2018)

[10] D. Fargion, A. Aiello, and R. Conversano, Proceedings of the 26th ICRC, 1999. (1999)

[11] A. Letessier-Selvon, 2001 AIP Conf. Proc. 566 157, Preprint astro-ph/0009444, (2001)

[12] T. Sjöstrand, S. Mrenna and P. Skands, Comput. Phys. Comm. 178, 852, (2008)

[13] M. A. K. Glasmacher et al., Astroparticle Physics, 10 (1999) 291.

[14] F. Fenu et al. (Pierre Auger Collaboration), Proceedings of Science, ICRC2017 (2017) 486.

[15] M. Ackerman et al., arXiv:1903.04334

[16] A. Walker et al., Nucl. Instrum. Methods A, 301, (1991), 574.

[17] A. Borione et al., Nucl. Instrum. Methods A, 346, (1994), 329.

[18] C. Bonifazi et al., Nuclear Physics B - Proceedings Supplements, 190, (2009), 20.

[19] I. Tkachev, et al., Proceedings of the 31st ICRC Beijing ID, (2011), p. 1311.

[20] R. Abbassi et al., Nucl. Instrum. Methods A, 700, (2013), 188.

[21] The IceCube Collaboration, Proceedings of Science ICRC2017, (2017) 997.

[22] R. Enberg, M. H. Reno, I Sarcevic, Phys. Rev. D, 78 (2008), 043005.

[23] J. Abraham, et al., NIM A, 613, (2010), 29. 